\title{
Mamoplastia en gigantomastia con areolas divergentes
}

\section{Mammoplasty in macromastia with divergent areolas}

Olímpio Aguiar, P.*, Vieira da Silva J r. V.**, Rodrigues de Miranda Filho, A.**, Veras Aguiar, C.***

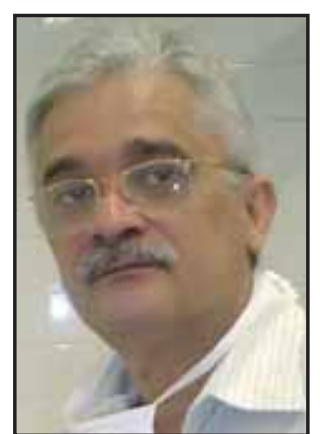

Olímpio Aguiar, P.
Resumen

El conocimiento anatómico de la mama, principalmente de su circulación e inervación, permiten un dominio en la manipulación del parénquima mamario y por consiguiente la creación de diversos tipos de colgajos de areola para la trasposición de la misma.

En el presente artículo, presentamos el caso de una mujer joven con mamilas muy divergentes en posición anatómica y con gigantomastia y ptosis bilateral. Los tiempos quirúrgicos empleados fueron los descritos por Pintaguy; no obstante, añadimos una modificación consistente en una incisión en línea recta en la dermis del borde lateral del área desepitelizada, respetando la vascularización y la inervación subdérmica.

Observamos que la técnica de Pitanguy asociada a la incisión dérmica en el borde lateral del colgajo de la areola permite su trasposición craneomedial en mamas extremadamente divergentes, resultando una mama con un aspecto estético más agradable y manteniendo su vascularización y sensibilidad.

Palabras clave Gigantomastia. Mamoplastia de reducción. Areolas.

Código numérico 52-5210

\begin{tabular}{|ll|}
\hline Key words & Mammary hipertrhophy. Reduction \\
& Mamoplasty. Areola. \\
Numeral Code & $52-5210$ \\
\hline
\end{tabular}




\section{Introducción}

Según Mojallal et al., la maniobra de desepidermización descrita por Schwartzmann en 1930 (1) y la creación de colgajos de areola supusieron un cambio importante en la evolución de la cirugía de la mama, al reducir la necesidad de utilizar injertos de areola en las reducciones mamarias (1). Schlenz et al. (2) refieren que el conocimiento anatómico de la mama, principalmente de su circulación e inervación, permiten un mayor dominio en la manipulación del parénquima mamario, lo que facilita la creación de colgajos de areola de varios tipos, como pueden ser el vertical proximal (Lassus) o el súperolateral (Skoog), o el vertical bipediculado (McKissock) o el horizontal transversal (Strömbeck) entre otros (2-6).

La modificación a la técnica de Arie (7), presentada por Pitanguy en 1959 (8) fue recibida con un gran entusiasmo, pues facilitó la cirugía de reducción mamaria en mamas de muy diversos tamaños. Actualmente sigue siendo una técnica muy empleada por su facilidad de aprendizaje (8).

Siguiendo a Cunha, la variedad de tamaños y formas de las mamas hace difícil la utilización de una técnica de mamoplastia de reducción aplicable a todos los tipos de mamas (9). Esa dificultad favorece la aparición de las más diversas maniobras para lograr ese fin (9). En el presente artículo, describimos una maniobra para la trasposición de las areolas en mamas en las que el complejo areola-pezón (CAP) se presenta muy lateralizado.

\section{Pacientes y métodos}

Presentamos el caso de una joven con areolas muy divergentes en posición anatómica, con gigantomastia y ptosis bilateral. No habíamos tenido anteriormente un caso que presentara unas areolas tan extremadamente divergentes. Esta característica añadía una dificultad mayor a la hora de programar la cirugía.

Tras el apropiado marcaje (Fig. 1) se practicó la reducción mamaria según la técnica de Pitanguy (10) siguiendo todos los tiempos propios de la misma. Sin embargo, se añadió como modificación una incisión en línea recta en la dermis del borde lateral del área desepitelizada, que respetaba la vascularización y la inervación subdérmicas (11-14) (Fig. 2). Solamente se cortaron las ramas nerviosas intradérmicas. Con esto, la areola se pedículo medialmente a través del tejido dermo-adiposo-glandular y lateralmente a través del tejido subdermo-adiposo-glandular. Además de la incisión dérmica se realizó una leve tracción del tejido incidido para favorecer su movilización.

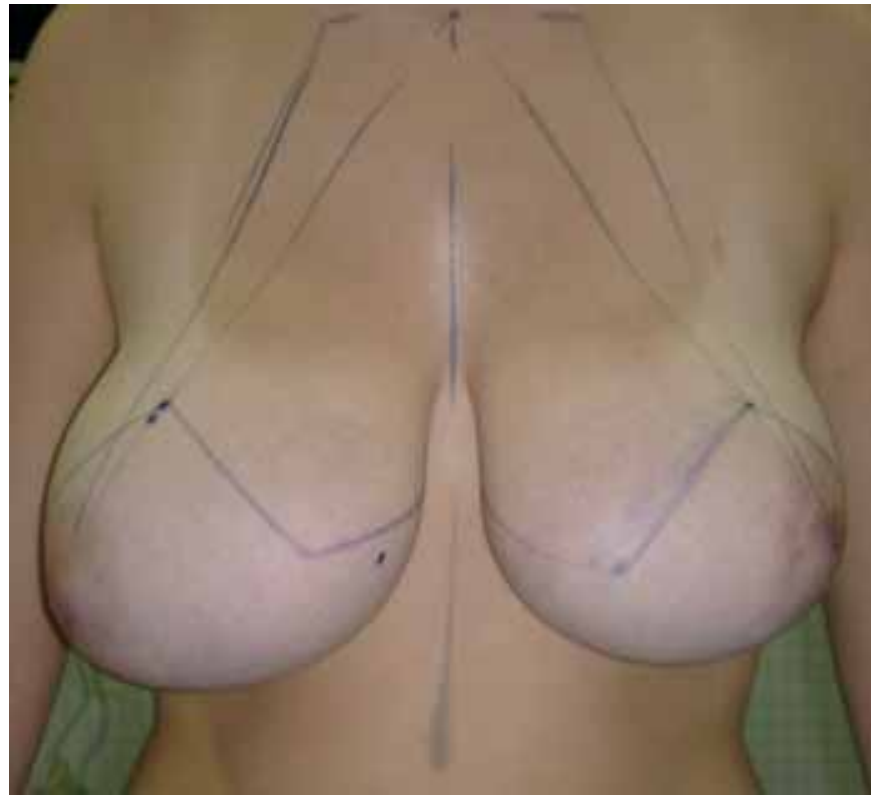

Fig. 1. Marcaje preoperatorio según técnica de Pitanguy.

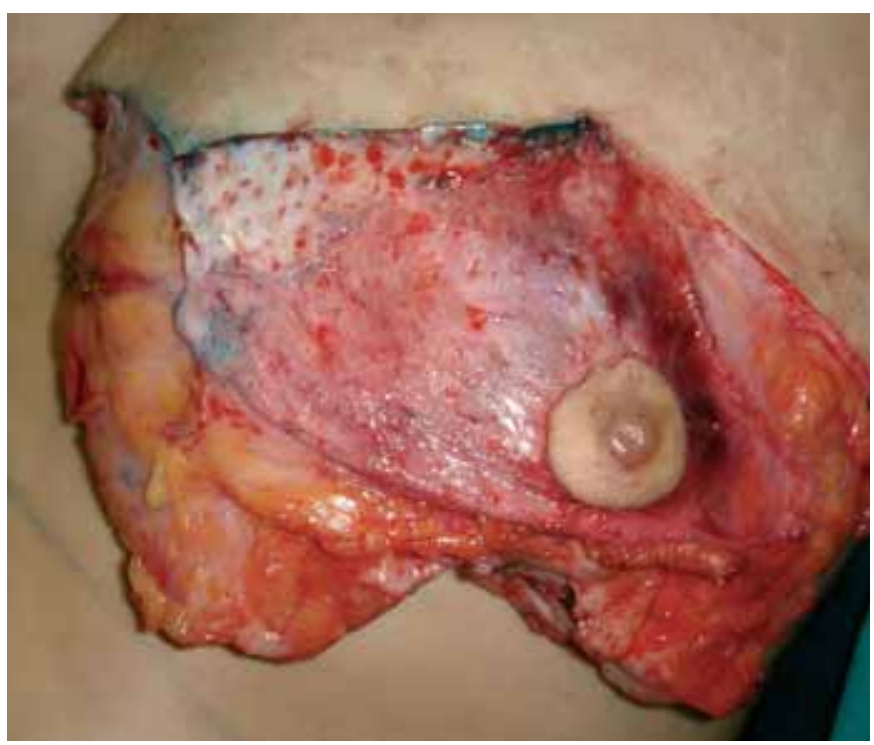

Fig. 2. Incisión en el borde lateral de la dermis desepitelizada.

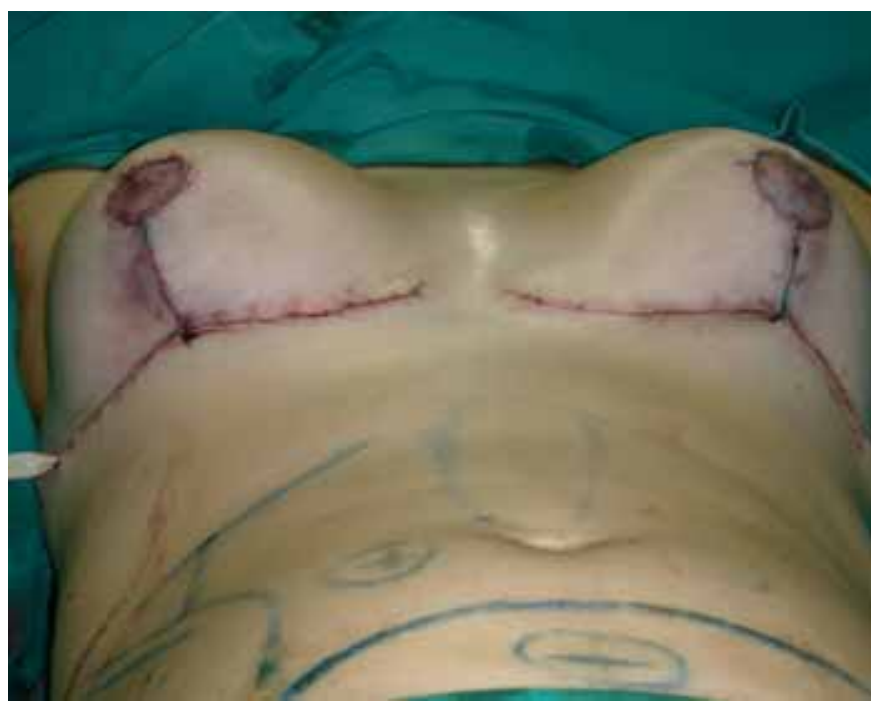

Fig. 3. Aspecto postoperatorio inmediato. Vemos la mama armada y suturada con cicatriz en T y con el CAP en posición más medial. 

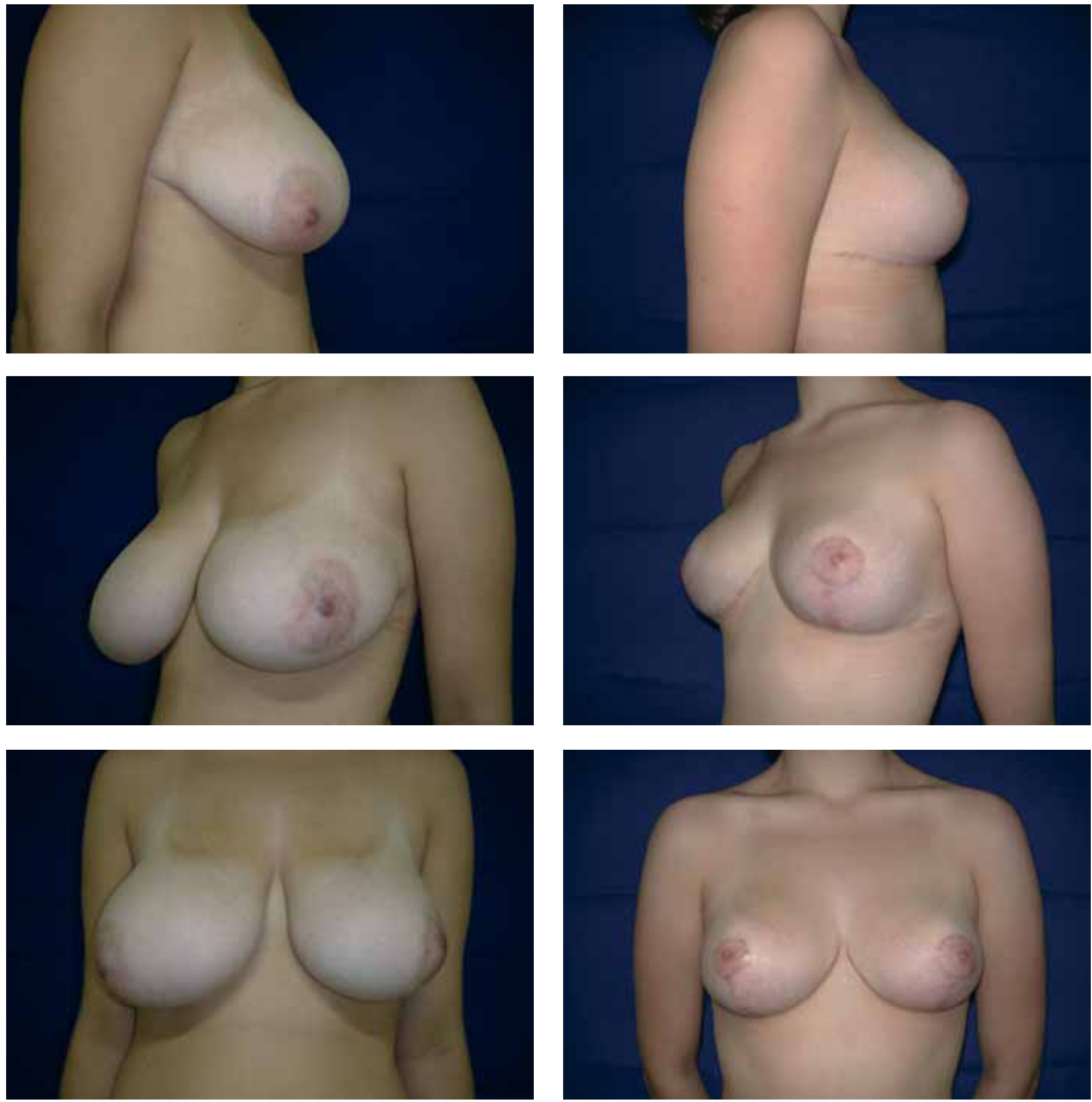

Fig. 4. Imagen comparativa del preoperatorio y del postoperatorio a los 3 meses de la intervención (Resección: mama derecha 540 gr., mama izquierda 520 gr.).

Logramos así una mayor facilidad en la trasposición de las areolas hasta su nueva posición, donde se fijaron sin tensión. A continuación las mamas fueron armadas y suturadas, resultando una cicatriz en $\mathrm{T}$, con un CAP más medial (Fig. 3).

\section{Resultados}

La maniobra descrita hizo posible una mayor libertad y seguridad en la trasposición del CAP hasta su nueva posición y sin tensión. Es por tanto una buena alternativa quirúrgica para un caso frecuentemente descrito en la literatura. La paciente evolucionó sin complicaciones de tipo isquemia o hematoma. El resultado obtenido fueron unas mamas con aspecto estético agradable tanto para el equipo quirúrgico y, lo más importante, para la paciente. No se produjeron alteraciones en la sensibilidad y se preservó la funcionalidad (Fig. 4).

Discusión

Estamos de acuerdo con Pitanguy acerca de la importancia sexual de las mama y de que cualquier 
técnica de mamoplastia de reducción debe mantener la fisiología glandular, garantizar una buena forma, minimizar la extensión de las cicatrices y asegurar la conservación de la sensibilidad (8).

Elegimos la técnica clásica de Pitanguy por su seguridad, puesto que mantiene la integridad vascular y nerviosa del CAP. Además permite un resultado estético armónico $(8,10)$.

Los estudios existentes sobre vascularización e inervación de la mama han permitido la mejora de las técnicas tradicionales y el nacimiento de otras nuevas que hacen posible resultados estéticos y funcionales más satisfactorios. Está demostrado que la circulación de la mama discurre a nivel intradérmico y subdérmico (2, 11-14); basándonos en este hecho, realizamos una incisión a lo largo de toda la extensión lateral de la dermis en el colgajo de areola.

Hidalgo (15), hace referencia a que la técnica en $\mathrm{T}$ invertida en la reducción mamaria continua siendo el mejor método para conseguir buenos resultados en diferentes tipos de gigantomastias. Permite seguridad y un buen abordaje para la resección glandular. Además, disminuye el potencial de aparición de cicatrices de mala calidad y posibilita lograr una forma mamaria y areolar más estética (15).

\section{Conclusiones}

Observamos que la técnica de Pitanguy asociada a una incisión subdérmica lateral en el colgajo de la areola permite una trasposición craneomedial en mamas extremadamente divergentes, logrando un aspecto estético agradable y conservando la vascularización y la sensibilidad.

\section{Dirección del autor}

Dr. Pedro Olimpío Aguiar

Instituto Dr. José Frota de Fortaleza (IJF)

Rua Barão do Rio Branco, 1816 - Centro

CEP: 60025-061

Fortaleza - CE - Brasil

\section{Bibliografía}

1. Mojallal A., Comparin J.P., Voulliaume D., Chichery A., Papalia I., e Foyatier J.-L: "Place de la réduction mammaire à pédicule supérieur dans lês gigantomasties." Ann. Chir. Plast. 2005; 50; 118.

2. Schlenz I., Rigel S., Schemper M., e Kuzbari R.: "Alteration of Nipple and Areola Sensitivity by Reduction Mammaplasty: A Prospective Comparison of Five Techniques." Plast. Reconstr. Surg. 2005; 115(3); 743.

3. Lassus C.: "A technique for breast reduction." Int. Surg. 1970; 53; 69.

4. Skoog T.: "A technique of breast reduction. Transposition of the nipple on a cutaneous vascular pedicle." Acta Chir. Scand. 1963; $126 ; 453$.

5. McKissock P.K.: "Reduction mamaplasty with a vertical dermal flap.” Plast. Reconstr. Surg. 1972; 49(3); 245.

6. Strömbeck J.D.: "Mammaplasty: report of a new technique base donw the two pedicle procedure." Brit. J. Plast. Surg. 1960; 13; 79.

7. Ariê G.: "Una nueva técnica de mastoplastia." Rev. Latinoamericana Cir. Plast. 1957; 3; 28.

8. Pitanguy I., Salgado F., e Radwansky H.N.: "Reduções mamárias - Técnicas pessoais sem descolamento cutâneo." Cirurgia Plástica fundamentos e arte: Cirurgia Estética. Vol. III. Editor José Marcos Mélega. Ed. Médica e Científica LTDA, Rio de Janeiro, 2003. Pp. 477-484.

9. Cunha R.J.C., Jaimovich C.A., Nogueira A.J.S., Lins D.S.M.R., e Nogueira C.F.: "Reduced mastoplasty: modified Silveira Neto's tactical maneuver for ascension of the nipple-areola complex." Arq. Bras. Med. Nav. 1990; 52(3); 65.

10. Pitanguy I.: "Une nouvelle technique de plastie mamaire. Étude de 245 cas consecutifs et presentation d'une technique personelle." Ann. Chir. Plast. 1962; 7;199.

11. Hefter W., Elvenes O.P., Lindholm P. "A retrospective quantitative assessment of breast sensation after lateral pedicle mammaplasty.” Br. J. Plast. Surg. 2003; 56; 667.

12. Schlenz I., Kuzbari R., Gruber H., Holle J.: "The sensitivity of the nipple-areola complex:ananatomic study." Plast. Reconstr. Surg. 2000; 105(3); 905.

13. Jaspars J.P., Posma A.N., van Immerseel A.A., e Gittenbergerde Groot A.C.: "The cutaneous innervation of the female breast and nipple - aréola complex: implications for surgery." Br. J. Plast. Surg. 1997; 50(4); 249.

14. Sarhadi N.S., Shaw Dunn J., Lee F.D., Soutar D.S.: “Anatomical study of the nerve supply of the breast, including the nipple and areola." Br. J. Plast. Surg. 1996; 49(3); 156.

15. Hidalgo D.A.: "Improving Safety and Aesthetic Results in Inverted T Scar Breast Reduction.” Plast. Reconstr. Surg. 1999; 103(3); 874. 


\title{
Mamoplastia em hipermastia com aréolas divergentes
}

\author{
Olímpio Aguiar, P.*, Vieira da Silva, J r. V.**, Rodrigues de Miranda Filho, A.**, Veras Aguiar, C.***
}

\section{Resumo}

O conhecimento anatômico da mama, principalmente de sua circulação e inervação, permitiu um domínio na manipulação do parênquima mamário, e isso permitiu a criação de retalhos areolados de vários tipos para a sua transposição. Neste artigo, é apresentado o caso de uma jovem que portava mamilos bem divergentes na sua posição anatômica comum e queixa de macromastia e ptose bilateral de mama. Os tempos cirúrgicos foram os mesmos descritos por Pitanguy. No entanto, a modificação acrescentada foi uma incisão em linha reta somente na derme da borda decorticada lateral, a qual respeitou a vascularização e a inervação subdérmica. Observou-se que a técnica de Pitanguy associada à incisão dérmica lateral no retalho areolado permitiu a transposição crâniomedial em mamas extremamente divergentes, pois resultou uma mama de aspecto estético agradável com a manutenção da sua vascularização e sensibilidade.

Palavras-chave: macromastia, mamoplastia, aréolas.

\section{Introdução}

Segundo Ali Mojallal et al, a manobra de desepitelização descrita por Schwartzmann em 1930 (1) e a confecção de retalhos areolados fizeram a cirurgia de mama sofrer uma mudança importante na sua trajetória, pois reduziu a indicação de enxertos de aréola nas reduções mamárias (1). Ingrid Schlenz et al. (2) referem que o conhecimento anatômico da mama, principalmente de sua circulação e inervação, permitiu um domínio na manipulação do parênquima mamário, e isso permitiu a criação de retalhos areolados de vários tipos, como o vertical proximal (Lassus), o súperolateral (Skoog), o vertical bipediculado (McKissock), o horizontal transversal (Strömbeck), entre outros (2-5).

A modificação da técnica de Arié (7), apresentada por Pitanguy em 1959 (8) foi recebida com grande entusiasmo, pois possibilitou a cirurgia de redução em mamas dos mais diversos tamanhos. Atualmente, ainda é uma técnica bastante empregada, principalmente, por sua facilidade de aprendizado (8).

Segundo Cunha, a variedade de tamanhos e formas de mamas torna difícil a utilização de uma única técnica para a mamoplastia redutora para todos os tipos de mamas (9). Essa dificuldade favorece o surgimento das mais diversas técnicas e manobras para esse fim (9). No presente artigo, é descrita uma manobra para a transposição das aréolas em mamas cujo complexo areolomamilar (CAM) apresentava-se bastante lateralizado.

\section{Pacientes e métodos}

Neste artigo, é apresentado o caso de uma jovem que portava mamilos bem divergentes na sua posição anatômica comum e queixa de macromastia e ptose bilateral de mama. Não tivemos oportunidade de caso parecido dada a raridade da situação de aréolas tão divergentes entre si. Tal característica tornava a programação cirúrgica uma dificuldade maior.

A paciente foi previamente marcada (Fig. 1) e teve as mamas ressecadas segundo a técnica de Pitanguy (10). Os tempos cirúrgicos foram os mesmos descritos por Pitanguy. No entanto, a modificação acrescentada foi uma incisão em linha reta somente na derme da borda decorticada lateral, a qual respeitou a vascularização e a inervação subdérmica (11-14) (Fig. 2). Portanto, foram incisados somente os ramos nervosos intradérmicos. Com isso, a aréola ficou pediculada medialmente por tecido dermoadiposograndular e lateralmente por tecido subdermoadiposoglandular. Além da incisão na derme foi realizada uma leve tração no tecido incisado para separar ainda mais a área decorticada da não-decorticada. Com isso, houve uma maior facilidade na transposição das aréolas para sua nova posição demarcada, onde permaneceu sem tensão.

A mama foi, então, armada e suturada, resultando numa cicatriz em T e num CAM mais medializado (Fig. 3).

\section{Resultados}

Esta manobra possibilitou uma maior liberdade e segurança na transposição do CAM para a sua nova posição, pois o mesmo apresentava-se sem tensão. Além disso, para esse caso incomum na literatura, ela mostrou-se como uma boa alternativa cirúrgica. A paciente evoluiu sem qualquer complicação de isquemia e hematoma. $\mathrm{O}$ resultado cirúrgico obtido foram mamas com aspecto estético agradável para a equipe cirúrgico e, principalmente, para a paciente. Não houve alteração de sensibilidade e a característica funcional foi preservada (Fig. 4).

\section{Discussão}

Segundo Pitanguy, pela importância sexual das mamas, a técnica de mamoplastia redutora deve manter a fisiologia grandular, garantir uma boa forma, reduzir a extensão das cicatrizes e assegurar a sensibilidade (8).

A técnica clássica de Pitanguy foi escolhida pela sua segurança, pois mantém a integridade vascular e nervosa do CAM. Além disso, permite um resultado estético mais gracioso $(8,10)$.

Os estudos sobre a vascularização e a inervação da mama têm permitido a inovação de técnicas antigas e o surgimento de novas que possibilitam resultados estéticos e funcionais satisfatórios. Eles demonstram que a vascularização do mamilo passa em nível subdérmico e a sensibilidade é garantida por ramos em nível intradérmico e subdérmico (2,11-14). Baseado nisso, foi realizada com segurança a incisão em toda a extensão lateral na derme do retalho areolado.

Hidalgo (15) refere que a técnica do $\mathrm{T}$ invertido na redução mamária permanece como o melhor método para conseguir bons resultados em diversos tipos de macromastias. Ela permite segurança e uma boa abordagem para a ressecção grandular. Além disso, diminui o potencial para a qualidade ruim da cicatriz e possibilita uma forma mamária e areolar mais estética (15).

\section{Conclusao}

Observou-se que a técnica de Pitanguy associada à incisão dérmica lateral no retalho areolado permitiu a transposição crâniomedial em mamas extremamente divergentes, pois resultou uma mama de aspecto estético agradável com a manutenção da sua vascularização e sensibilidade. 


\section{Comentario al trabajo uMamoplastia en gigantomastia con areolas divergentes॥}

\section{Dra. Manuela Berrocal R. Cirujano Plástico. Cartagena de Indias. Colombia. Presidenta del Consejo Consultivo de la FILACP.}

Fue un placer recibir el artículo de los colegas del Instituto de Cirugía Plástica Dr. José Frota, de Fortaleza-Caerá, Brasil, y comprobar que muchos colegas brasieliros, siguen permanentemente activos en su creatividad, tratando de buscar alternativas de solución a los problemas frecuentes de la patología mamaria.

Evidentemente el colgajo de pedículo lateral, es una alternativa excelente para corregir los problemas de malposición del complejo areolo-mamilar, por su viabilidad vascular e inervación, siendo descrito por autores franceses en el pasado. Más recientemente el Dr. L. Cárdenas -Camarena de Guadalajara, México, publicó un artículo en el American Journal de Marzo 2001, titulado: "Reduction mammoplasty with Superior Lateral Dermoglan- dular Pedicle: Another Alternativ", cuya diferencia radica en que el pedículo es lateral externo y el de los autores de este trabajo es lateral medial.

En la Revista Iberolatinoamericana, Vol 26 - No 3 de 2000, en un trabajo de mi autoría, página 235 , aparecen las fotografías de un caso similar al de los autores, corregido con un colgajo de pedículo superior, cuya mayor diferencia en relación con las otras técnicas radica en el tamaño de la cicatriz en J, aplicada por la autora en grandes hipertrofias y gigantomastias.

Mis sinceras felicitaciones a los autores por seguir demostrando que el talento Iberolatinoamericano de la Cirugía Plástica, sigue generando y transformando el conocimiento científico.

\section{Respuesta al comentario de la Dra. Manuela Berrocal R.}

\section{Dr. Vieira da Silva, J r. V.}

Antes de mais nada, gostaríamos de agradecer a gentileza e os elogios recebidos da Dra. Manuela Berrocal a cerca do nosso trabalho. É de grande honra para nós poder compartilhar nossa experiência com pessoas tão responsáveis e competentes à profissão.

Gostaríamos de esclarecer a idéia do nosso retalho. Inicialmente, no retalho de pedículo lateral, o retalho é incisado em todos os planos na porção medial e ele fica pediculado de forma dermoglandular lateral. Nosso retalho não é incisado em todos os planos na sua porção lateral. Fazemos apenas uma incisão superficial na derme e uma leve tração do tecido para deixá-lo mais frouxo. Pela incisão é possível observar alguns ramos vasculares na subderme. Com isso, o retalho passa a ser pediculado dermoglandular na sua porção medial e lipoglandular na sua porção lateral. Isso torna esse retalho ainda mais seguro, pois mantêm preservados os ramos vasculares e nervosos subdérmicos do complexo areolomamilar (CAM) na sua porção lateral, além daqueles da sua porção medial, diferentemente da técnica descrita para pedículo dermoglandular lateral, que preserva apenas da porção lateral. Além disso, esse retalho não permite uma medialização tão ampla como a proposta por nossa manobra.

No caso da paciente citada, o CAM era muito lateralizado e precisávamos de uma manobra para permitir sua ampla medialização e sem tensão. Por nossa experiência, achamos de grande risco tentar fazer um retalho areolado de pedículo superior pela possibilidade de manter-se um retalho muito tenso e o retalho lateral não permitia uma medialização tão ampla para este caso." 